\title{
Flat Space Cosmology as a Model of Light Speed Cosmic Expansion-Implications for the Vacuum Energy Density
}

\author{
Eugene Terry Tatum ${ }^{1}$, U. V. S. Seshavatharam² \\ ${ }^{1} 760$ Campbell Ln. Ste. 106 \#161, Bowling Green, KY, USA \\ ${ }^{2}$ Honorary Faculty, I-SERVE, S. No-42, Hitex Road, Hitech City, Hyderabad, India \\ Email: ett@twc.com, seshavatharam.uvs@gmail.com
}

How to cite this paper: Tatum, E.T. and Seshavatharam, U.V.S. (2018) Flat Space Cosmology as a Model of Light Speed Cosmic Expansion-Implications for the Vacuum Energy Density. Journal of Modern Physics, 9, 2008-2020.

https://doi.org/10.4236/jmp.2018.910126

Received: August 22, 2018

Accepted: September 10, 2018

Published: September 13, 2018

Copyright (C) 2018 by authors and Scientific Research Publishing Inc. This work is licensed under the Creative Commons Attribution International License (CC BY 4.0).

http://creativecommons.org/licenses/by/4.0/

\begin{abstract}
Cosmologists have long ignored a stipulation by quantum field theorists that the vacuum pressure $p$ corresponding to the zero-state vacuum energy must always be equal in magnitude to the vacuum energy density $\rho$ (i.e., $p=\rho)$. Although general relativity stipulates the additional condition of proportionality between the vacuum gravitational field and $(\rho+3 p)$, the equation of state for the cosmic vacuum must fulfill both relativistic and quantum stipulations. This paper fully integrates Flat Space Cosmology (FSC) into the Friedmann equations containing a cosmological term, with interesting implications for the nature of dark energy, cosmic entropy and the entropic arrow of time. The FSC vacuum energy density is shown to be equal to the cosmic fluid bulk modulus at all times, thus meeting the quantum theory stipulation of $(p=\rho)$. To date, FSC is the only viable dark energy cosmological model which has fully-integrated general relativity and quantum features.
\end{abstract}

\section{Keywords}

Cosmology Theory, General Relativity, Dark Energy, Cosmic Flatness, Cosmic Entropy, Entropic Arrow of Time, Cosmic Inflation, Milne Universe, Black Holes, Cosmological Constant Problem

\section{Introduction and Background}

Flat Space Cosmology (FSC) is a mathematical model of universal expansion which has proven to be remarkably accurate in comparison to observations [1] [2] [3] [4]. FSC began as a heuristic model following the Penrose-Hawking idea of treating the expanding universe as a time-reversed giant black hole, which is 
smoothly expanding as opposed to smoothly collapsing [5] [6]. However, we have also recently proven it to be a general relativity model by successfully integrating the FSC assumptions into the Friedmann equations which include a cosmological term and a global curvature term $k$ set to zero [7] [8]. The relevant equations will be repeated in this paper for clarity.

One of the results of integrating FSC into the Friedmann equations is that the following relation holds true in FSC:

$$
\frac{3 H^{2} c^{2}}{8 \pi G} \cong \frac{\Lambda c^{4}}{8 \pi G}
$$

This is merely a reflection that global space-time in FSC is flat during the cosmic expansion. As stipulated by the space-time curvature rules of general relativity, a globally flat universe must have a net energy density of zero. Otherwise, if the positive energy density and negative energy density terms were not equal in magnitude, there would be an observable global space-time curvature representative of the greater energy density term.

Astronomical observations [9] [10] [11], in the context of general relativity, indicate that a mysterious energy presumably within the cosmic vacuum must be exerting a force in opposition to that of attractive gravity. Thus, this vacuum "dark energy" is defined as a negative energy with respect to the positive energy of cosmic matter. General relativity stipulates the associated vacuum gravitational field to be proportional to $(\rho+3 p)$. Quantum field theory makes the additional stipulation that the vacuum pressure $p$ corresponding to the zero-state vacuum energy must always be equal in magnitude to the vacuum energy density $\rho$ (i.e., $p=\rho$ ). Cosmologists, who seem to be particularly focused on $(\rho+3 p)$, appear not to be strictly adhering to the quantum theory stipulation. If need be, they appear willing to consider an equation of state $w$ value other than exactly minus one. Nevertheless, the cosmic vacuum equation of state must follow both relativistic and quantum stipulations.

The purpose of this paper is to show how the FSC Friedmann equations evolve further from Equation (1) and what they imply, especially with respect to the vacuum energy conditions stipulated by general relativity and quantum field theory. Before doing so, however, it is useful to review the five current assumptions of FSC and its observational correlations.

\subsection{The Five Assumptions of Flat Space Cosmology}

1) The cosmic model is an ever-expanding sphere such that the cosmic horizon always translates at speed of light $c$ with respect to its geometric center at all times $t$. The observer is operationally defined to be at this geometric center at all times $t$.

2) The cosmic radius $R_{t}$ and total matter mass $M_{t}$ follow the Schwarzschild formula $R_{t} \cong 2 G M_{t} / c^{2}$ at all times $t$.

3) The cosmic Hubble parameter is defined according to $H_{t} \cong c / R_{t}$ at all times $t$. 
4) Incorporating our cosmological scaling adaptation of Hawking's black hole temperature formula, at any radius $R_{t}$ cosmic temperature $T_{t}$ is inversely proportional to the geometric mean of cosmic total matter mass $M_{t}$ and the Planck mass $M_{p l} . R_{p l}$ is defined as twice the Planck length (i.e., as the Schwarzschild radius of the Planck mass black hole). With subscript $t$ for any time stage of cosmic evolution and subscript $p l$ for the Planck scale epoch, and incorporating the Schwarzschild relationship between $M_{t}$ and $R_{t}$,

$$
\begin{aligned}
& k_{B} T_{t} \cong \frac{\hbar c^{3}}{8 \pi G \sqrt{M_{t} M_{p l}}} \cong \frac{\hbar c}{4 \pi \sqrt{R_{t} R_{p l}}} \\
& \left\{\begin{array}{l}
M_{t} \cong\left(\frac{\hbar c^{3}}{8 \pi G k_{B} T_{t}}\right)^{2} \frac{1}{M_{p l}} \\
R_{t} \cong \frac{1}{R_{p l}}\left(\frac{\hbar c}{4 \pi k_{B}}\right)^{2}\left(\frac{1}{T_{t}}\right)^{2} \\
R_{t} T_{t}^{2} \cong \frac{1}{R_{p l}}\left(\frac{\hbar c}{4 \pi k_{B}}\right)^{2} \\
H_{t} \cong \frac{c}{R_{t}}
\end{array}\right.
\end{aligned}
$$

5) Total entropy of the cosmic model follows the Bekenstein-Hawking black hole entropy formula [12] [13]

$$
S \cong \frac{\pi R^{2}}{L_{p}^{2}}
$$

The first two assumptions are based upon a literal interpretation of the Hawking-Penrose conjecture as it would pertain to a smoothly-expanding Schwarzschild black hole. The third assumption (Hubble parameter) treats maximally redshifted radial photons at the cosmic model horizon as moving with speed of light $c$ relative to the geometric center at a distance of horizon radius $R_{t}$. This is a stipulation of relativity. The fourth assumption is a cosmic temperature scaling assumption. While it shows similarity to the static Hawking black hole temperature formula, the FSC cosmic model is treated as scaling in Planck mass increments. This allows for dynamic cosmic expansion modeling from the Planck scale epoch. Finally, the fifth assumption utilizes the Bekenstein-Hawking entropy definition, which seems appropriate for a model of the Hawking-Penrose conjecture. The numerous observational correlations of FSC are given, most recently, in "Temperature Scaling in Flat Space Cosmology in Comparison to Standard Inflationary Cosmology" [7] and "Clues to the Fundamental Nature of Gravity, Dark Energy and Dark Matter” [8].

As previously reported [Tatum, et al. (2015)], a number of past, current and future cosmological parameters can be calculated using the FSC model. The accuracy of these correlations with observations is largely accomplished by incorporating the appropriate cosmological scaling formula for cosmic temperature [see the top equation in relation (2)]. This equation, by incorporating elementa- 
ry and fundamental constants of nature, allows for FSC scaling from the Planck scale to the current scale. Thus, in this sense, FSC can be considered a quantum cosmology model.

\subsection{Cosmological Parameter Derivations of FSC}

Incorporation of the FSC assumptions into the cosmological scaling temperature formula allows for the following cosmological parameter definitions. Current observational parameters are calculated in the right-hand column. The only free parameter in any of these equations is the cosmic temperature. The currently observed cosmic temperature value: $\mathrm{T}_{0}=2.72548{ }^{\circ} \mathrm{K}$.

$$
\begin{aligned}
& R \cong \frac{\hbar^{3 / 2} c^{7 / 2}}{32 \pi^{2} k_{B}^{2} T^{2} G^{1 / 2}} \quad R_{0} \cong \frac{\hbar^{3 / 2} c^{7 / 2}}{32 \pi^{2} k_{B}^{2} T_{0}^{2} G^{1 / 2}} \\
& H \cong \frac{32 \pi^{2} k_{B}^{2} T^{2} G^{1 / 2}}{\hbar^{3 / 2} c^{5 / 2}} \quad H_{0} \cong \frac{32 \pi^{2} k_{B}^{2} T_{0}^{2} G^{1 / 2}}{\hbar^{3 / 2} c^{5 / 2}} \\
& t \cong \frac{\hbar^{3 / 2} c^{5 / 2}}{32 \pi^{2} k_{B}^{2} T^{2} G^{1 / 2}} \quad t_{0} \cong \frac{\hbar^{3 / 2} c^{5 / 2}}{32 \pi^{2} k_{B}^{2} T_{0}^{2} G^{1 / 2}} \\
& M \cong \frac{\hbar^{3 / 2} c^{11 / 2}}{64 \pi^{2} k_{B}^{2} T^{2} G^{3 / 2}} \quad M_{0} \cong \frac{\hbar^{3 / 2} c^{11 / 2}}{64 \pi^{2} k_{B}^{2} T_{0}^{2} G^{3 / 2}} \\
& M c^{2} \cong \frac{\hbar^{3 / 2} c^{15 / 2}}{64 \pi^{2} k_{B}^{2} T^{2} G^{3 / 2}} \quad M_{0} c^{2} \cong \frac{\hbar^{3 / 2} c^{15 / 2}}{64 \pi^{2} k_{B}^{2} T_{0}^{2} G^{3 / 2}} \\
& H_{0}=2.167862848658891 \times 10^{-18} \mathrm{~s}^{-1}\left(66.89325791854758 \mathrm{~km} \cdot \mathrm{s}^{-1} \cdot \mathrm{Mpc}^{-1}\right)
\end{aligned}
$$

This derived current Hubble parameter value fits very closely with the low end range of the 2015 Planck Collaboration consensus observational value of 67.8 $+/-0.9 \mathrm{~km} \cdot \mathrm{s}^{-1} \cdot \mathrm{Mpc}^{-1}$.

$$
t_{0} \cong \frac{1}{H_{0}}=4.612837941379141 \times 10^{17} \mathrm{~s}\left(14.61694683819266 \times 10^{9} \text { sidereal } \mathrm{yrs}\right)
$$

(multiplying by 1 sidereal yr/3.155814954 $\times 10^{7} \mathrm{~s}$ )

This value is the reciprocal of the derived Hubble parameter, as one would expect for a flat space-time cosmic model in comparison to the standard inflationary model. 13.7 billion years is now consensus for the standard model.

$$
R_{0} \cong \frac{c}{H_{0}}=1.382894024801713 \times 10^{26} \mathrm{~m}\left(14.61720137583068 \times 10^{9} \text { light-yrs }\right)
$$

(multiplying by 1 Julian light-yr/9.4607304725808 $\times 10^{15} \mathrm{~m}$ )

This current cosmic radius value correlates with current cosmic time by $R_{o}=$ $c t_{0}$. For reasons given in the seminal FSC papers, a perpetually flat and finite space-time cosmology model has no need to incorporate a superluminal inflationary mechanism to solve the flatness and horizon problems.

$$
V_{o l}=\frac{4 \pi}{3}\left(\frac{c}{H_{0}}\right)^{3}=1.107784564915062 \times 10^{79} \mathrm{~m}^{3}
$$




$$
M_{0}=\frac{c^{3}}{2 G H_{0}}=9.311265291518025 \times 10^{52} \mathrm{~kg}
$$

This total matter mass number can be compared very favorably to a rough estimate made from astronomical observations. The visible matter consists of roughly 100 billion galaxies averaging roughly 100 billion stars each, of average star mass equal to roughly $1.4 \times 10^{30} \mathrm{~kg}$ (70 percent of solar mass), totaling to roughly $1.4 \times 10^{52} \mathrm{~kg}$. The 2015 Planck Collaboration report indicates a universal matter ratio of approximately 5.47 parts dark matter to 1 part visible (baryonic) matter. This brings the total estimated matter in the observable universe to approximately $9.1 \times 10^{52} \mathrm{~kg}$. A recent study [14] of average mass density of intergalactic dust gives a value of approximately $10^{-30} \mathrm{~kg} \cdot \mathrm{m}^{-3}$. Since this is approximately 1 part intergalactic dust to 1000 parts galactic and perigalactic matter, intergalactic dust does not appreciably modify the total observational estimated mass of matter given above. Accordingly, this observational estimate is remarkably close to the above FSC theoretical calculation of total cosmic matter mass. By the FSC Friedmann equations (below), the positive matter mass-energy must always be equal in absolute magnitude to the negative dark energy. This predicts a 50/50 cosmic energy density percentage ratio as opposed to the approximately 30/70 ratio currently claimed by standard cosmology proponents. However, without unequivocally proving cosmic acceleration, standard cosmology cannot yet rightfully claim this 30/70 ratio (see Discussion section).

$$
\begin{gathered}
M_{0} c^{2}=\frac{c^{5}}{2 G H_{0}}=8.368547901344209 \times 10^{69} \mathrm{~J} \\
\rho_{0}=\frac{3 H_{0}^{2}}{8 \pi G}=8.405303329200976 \times 10^{-27} \mathrm{~kg} \cdot \mathrm{m}^{-3} \quad \text { (critical mass density) }
\end{gathered}
$$

This closely approximates the observational critical density.

$$
\rho_{0} c^{2}=\frac{3 H_{0}^{2} c^{2}}{8 \pi G}=7.554309895973191 \times 10^{-10} \mathrm{~J} \cdot \mathrm{m}^{-3}
$$

This closely approximates the observational critical energy density and the observational vacuum energy density. They are equal in absolute magnitude in FSC.

\section{Flat Space Cosmology Friedmann Equations}

With respect to the Friedmann equations, those incorporating a non-zero cosmological term (i.e., a dark energy term) are now the most relevant since the 1998 Type Ia supernovae discoveries. Therefore, accepting Friedmann's starting assumptions of homogeneity, isotropism and an expanding cosmic system with a stress-energy tensor of a perfect fluid, we have his cosmological equation

$$
\frac{\dot{a}^{2}+k c^{2}}{a^{2}} \cong \frac{8 \pi G \rho+\Lambda c^{2}}{3}
$$

This equation is derived from the 00 (i.e., energy density) component of the Einstein field equations. Since the global curvature term $k$ is always zero in FSC, 
Equation (9) reduces to

$$
\left(\frac{\dot{a}}{a}\right)^{2} \cong H^{2} \cong \frac{8 \pi G \rho}{3}+\frac{\Lambda c^{2}}{3}
$$

With rearrangement, we have

$$
\frac{3 H^{2}}{8 \pi G}-\frac{\Lambda c^{2}}{8 \pi G} \cong \rho
$$

This is the relevant Friedmann equation for cosmic mass density. Multiplying all terms by $c^{2}$ gives us the relevant Friedmann equation for cosmic energy density

$$
\frac{3 H^{2} c^{2}}{8 \pi G}-\frac{\Lambda c^{4}}{8 \pi G} \cong \rho c^{2}
$$

At this point it is crucial to remember that Friedmann's energy density derivation of Einstein's field equations for the cosmic system as a whole (i.e., globally) can be interpreted in the form of additive space-time curvatures represented by the individual terms. The first term can be read as the positive energy density (i.e., the positive space-time curvature) term; the second term can be read as the negative energy density (i.e., the negative space-time curvature) term; and the third term can be read as the summation (i.e., net) energy density term for global cosmic space-time curvature. Since global space-time is treated as constantly and perfectly flat in FSC, the third term must always have a net value of zero energy density. This is entirely in keeping with the general theory of relativity, as applied to cosmology, as well as current cosmological observations of flatness (i.e., critical density). Hence, in FSC

$$
\frac{3 H^{2}}{8 \pi G} \cong \frac{\Lambda c^{2}}{8 \pi G}
$$

And

$$
\frac{3 H^{2} c^{2}}{8 \pi G} \cong \frac{\Lambda c^{4}}{8 \pi G}
$$

From these respective critical mass density and energy density equations, it is obvious that the FSC model defines the Lambda term $\Lambda$ by

$$
\Lambda \cong \frac{3 H^{2}}{c^{2}}
$$

In FSC and other realistic linear Milne-type models, Hubble parameter $H$ is a quantity which scales with cosmic time and is defined as

$$
H \cong \frac{c}{R}
$$

where $c$ is the speed of light and $R$ is the cosmic radius as defined by the Schwarzschild formula

$$
R \cong \frac{2 G M}{c^{2}}
$$

where $M$ represents the total matter mass of the cosmic system and $G$ is the uni- 
versal gravitational constant. Therefore, FSC Equation (15) substituted by Equation (16) gives

$$
\Lambda \cong \frac{3}{R^{2}}
$$

So the Lambda term $\Lambda$ is also a scalar quantity (i.e., like the Hubble parameter, not actually a constant) over the great span of cosmic time. This indicates that FSC is a dynamic dark energy quintessence model.

Crucially, Equation (18) allows one to compare the Lambda term $\Lambda$ with total entropy for the FSC cosmic system over the span of cosmic time. Recalling the Bekenstein-Hawking derivation of black hole entropy [Bekenstein (1974); Hawking (1976)] as directly proportional to the event horizon surface area $\left(4 \pi R^{2}\right)$, we can apply their formula for cosmic entropy

$$
S \cong \frac{\pi R^{2}}{L_{p}^{2}}
$$

Then substituting Equation (18) into Equation (19) and rearranging terms

$$
\Lambda \cong \frac{3 \pi}{S L_{p}^{2}}
$$

Thus, the Lambda term $\Lambda$ in FSC is inversely proportional to total cosmic entropy $S$ at all times. Substituting Equation (20) into Equation (15) gives

$$
S \cong \frac{\pi c^{2}}{H^{2} L_{p}^{2}}
$$

and

$$
H \cong \frac{c}{L_{p}} \sqrt{\frac{\pi}{S}}
$$

And, since the reciprocal of the Hubble parameter is the measure of cosmic time $t$ in FSC

$$
t \cong \frac{L_{p}}{c} \sqrt{\frac{S}{\pi}}
$$

So cosmic time is always directly proportional to $\sqrt{S}$, with entropy $S$ as defined by Bekenstein and Hawking. Thus, the "entropic arrow of time" is clearly defined in the FSC model.

The dark energy density cosmological term is not only expressed as $\left(\Lambda c^{4} / 8 \pi G\right)$ in FSC Friedmann equation (14) but, by incorporating equation (20) into this term, we now have a dark energy density equation

$$
\frac{\Lambda c^{4}}{8 \pi G} \cong \frac{3 c^{4}}{8 G S L_{p}^{2}} \cong \frac{3 H^{2} c^{2}}{8 \pi G}
$$

wherein any of these terms can be used interchangeably to quantify the absolute magnitude of the cosmic dark energy density at all times.

Since the FSC cosmic expansion model follows the Friedmann starting assumptions of homogeneity, isotropism, and an expanding cosmic system with a 
stress-energy tensor of an ideal fluid, one can consider the bulk modulus $B$ of classical mechanics to apply to such a fluid. Thus, the wave velocity $v_{w}$ of the cosmic fluid should have the following relationship

$$
v_{w}=\sqrt{\frac{B}{d}}
$$

In a realistic cosmic model, where $v_{w}=c$, and $d=p$, and $B$ is the cosmic vacuum bulk modulus, this can be expressed as

$$
c=\sqrt{\frac{B}{p}}
$$

Thus,

$$
B=p c^{2}
$$

Therefore, the pressure of this particular ideal fluid (the cosmic vacuum) must always equal its energy density by virtue of the fact that the cosmic vacuum always has a wave velocity of $c$. This satisfies the quantum theory $(p=\rho)$ stipulation for the zero-state vacuum energy.

\section{Discussion}

Cosmologists have long ignored a stipulation by quantum field theorists that the vacuum pressure $p$ corresponding to the zero-state vacuum energy must always be equal in absolute magnitude to the vacuum energy density $\rho$ (i.e., $p=\rho$ ). While general relativity further stipulates proportionality between the repulsive gravity field of dark energy and $(\rho+3 p)$, there appears to be little interest within the standard inflationary cosmology community concerning strict adherence to the above quantum theory stipulation. Unfortunately, the effect of ignoring the quantum field stipulation is that it becomes difficult for cosmologists to otherwise explain an observed equation of state term $w=-1.006+/-0.045$ (Planck 2015). Whereas, a value of exactly -1.0 is a prediction of the FSC model, as is obvious from equation (27), when adopting the convention of opposite pressure and density signages.

The discovery of dark energy within the cosmic vacuum applies to cosmic models an accelerator pedal in opposition to the brake pedal of attractive gravity. An important question yet to be resolved is whether the cosmic acceleration value is precisely zero or very slightly positive. Deep statistical analysis of the Supernova Cosmology Project compilation data shows only two remaining viable categories of dark energy cosmological models: realistic Milne-type $R_{h}=c t$ models (with a zero acceleration value, by definition); and the standard model (with an exceedingly small positive acceleration value). A number of very recent papers [15] [16] [17] [18] show strong statistical support for the continued viability of realistic Milne-type $R_{h}=c t$ models.

The FSC model is the most realistic Milne-type $R_{h}=c t$ model to date, by virtue of the fact that it is now fully integrated into the Friedmann equations, as shown in this paper, and shows tight correlations with the 2015 Planck Collabo- 
ration report findings, including their consensus observational Hubble parameter value of $67.8+/-0.9 \mathrm{~km} \cdot \mathrm{s}^{-1} \cdot \mathrm{Mpc}^{-1}$. A realistic Milne-type model, in sharp contrast to Milne's original "empty universe" model [19], is one which contains gravitational matter. As further evidence of the continued viability of realistic $R_{h}$ $=c t$ models, the following open source graph from the Supernova Cosmology Project [20] is shown in Figure 1.

The FSC realistic Milne-type $R_{h}=c t$ model is mathematically a perpetual critical density (i.e., "flat") model, as shown in the seminal FSC papers [Tatum, et al. (2015)]. Therefore, it falls on the "Flat" universe model line in Figure 1.

Those with knowledge of the observational studies of the ratio of dark matter to visible matter realize the difficulty of determining a precise co-moving value for this ratio at the present time. Too little is yet known about dark matter for such precision. Galactic and perigalactic distributions of dark matter can be surprisingly variable, as evidenced by the 29 March 2018 report in Nature [21] of a galaxy apparently completely lacking in dark matter! Although the 2015 Planck Collaboration consensus is a large-scale approximate ratio of 5.47 parts dark matter to 1 part visible matter, this can only be a rough estimate of the actual co-moving ratio, particularly if this ratio varies significantly over cosmic time. A 10-to-1 actual ratio in co-moving galaxies remains a possibility, and would change the actual ratio of total matter mass-energy to dark energy to essentially unity (i.e., 50\% matter and 50\% dark energy). The intersection zone of tightest constraints shown in Figure 1 would then correlate with 0.5 Omega_ matter and 0.5 Omega_Lambda. This is one of several important testable predictions discriminating the FSC model from the standard model.

So long as these models are in a statistical dead heat, it is reasonable to ask if one of the two models is more compatible with the quantum theory stipulation of equality between the vacuum pressure and the vacuum energy density. The answer to this question is clearly in favor of FSC, because the predicted 50/50 percentage ratio for FSC meets the quantum theory stipulation, whereas the standard model 30/70 percentage ratio does not.

The reason why the 50/50 percentage ratio of FSC implies equality between the vacuum pressure and the vacuum energy density (and why the standard model $30 / 70$ percentage ratio does not imply such equality) is contained in the final four equations of this paper [Equations (24) thru (27)]. The crucial equations are (24) and (27). Only in the FSC model can the vacuum energy density $\left(\Lambda c^{4} / 8 \pi G\right)$ be shown to be equal in absolute magnitude to both the total matter mass-energy density $\left(3 H^{2} c^{2} / 8 \pi G\right)$ and the vacuum pressure represented by the bulk modulus $B$ term in Equation (27).

One of the unexpected findings of integrating the FSC model into the Friedmann equations is the intriguing discovery of a possible close relationship between dark energy and total cosmic entropy [see relations (18) through (24)]. A review of prior publications concerning this possible relationship indicates that theoretical physicist Roger Penrose explores this subject in great detail in his recent book entitled "Fashion Faith and Fantasy in the New Physics of the 


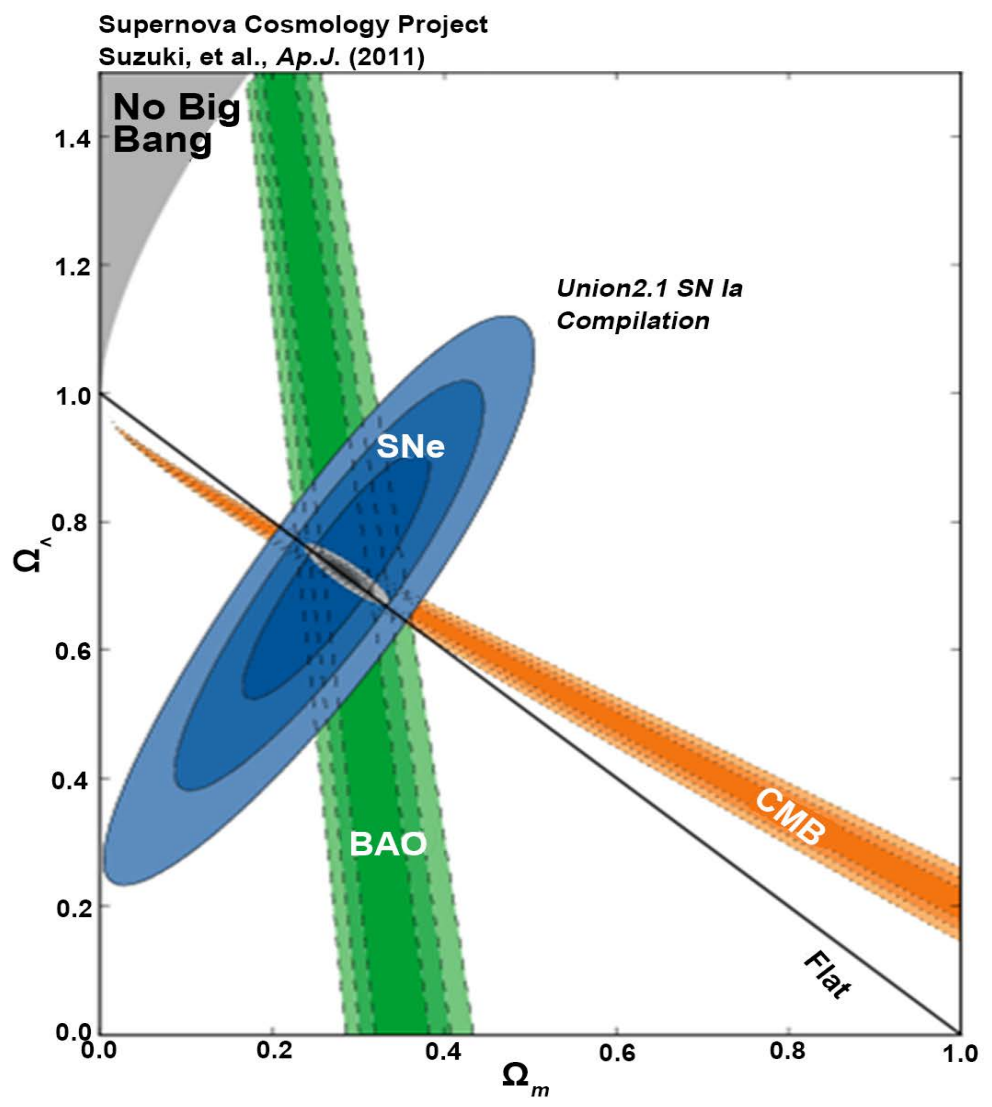

Figure 1. Accumulated Supernova ( $\mathrm{SNe}$ ), BAO and $\mathrm{CMB}$ data with highlighted constraints.

Universe" [22] (pages 275-285). Perhaps most notably, Penrose uses the Bekenstein-Hawking black hole entropy formula to derive the same equation (in rearranged form, on his page 277) as FSC relation (18) in this paper. This gives us great confidence that resulting FSC Equations (19) through (24), showing mathematical relationships between total cosmic entropy and the Hubble parameter, cosmic time ("entropic arrow of time") and vacuum energy density, are indeed realistic and correct. Thus, FSC also may prove to be a useful cosmological model correlate to Erik Verlinde's "entropic gravity" [23] and "emergent gravity" [24] theories.

It is important to reiterate that the equation of state for the cosmic vacuum is stipulated by both relativistic and quantum considerations. By apparently ignoring the stipulation from quantum field theory that $(p=\rho)$ must always hold true for the cosmic vacuum, standard cosmology practitioners appear to have reached an impasse between general relativity and quantum theory. The FSC model, on the other hand, has fully integrated these relativistic and quantum stipulations, while maintaining remarkable accuracy with respect to current observations.

\section{Summary and Conclusions}

The FSC model of cosmology was developed as a heuristic mathematical model 
of the Hawking-Penrose idea that an expanding universe arising from a singularity state can be modeled as a time-reversed giant black hole. This idea was an extension of Penrose's paper [Penrose (1965)] on the singularities of black holes and cosmology. Hawking's doctoral thesis took the idea further by proving the validity of time-reversal in the treatment of general relativity as it concerns cosmology [Hawking and Penrose (1970)]. Finally, the FSC model completes this idea by incorporating scaling black hole equations suitable for cosmology. Thus, the proven accuracy of FSC with respect to current astronomical observations does not appear to be an accident.

To overcome any potential objections that FSC does not fit within general relativity, this paper fully integrates FSC into the Friedmann equations. The implications of the FSC Friedmann equations offer unique insights into the possible nature of dark energy with respect to total cosmic entropy and the entropic arrow of time (see especially FSC reference [8]).

The primary purpose of this paper concerns the vacuum energy density implications of the FSC Friedmann equations. Particular attention is paid to Equation (1) as it relates to Equations (24) thru (27). Since a perpetually flat universe model implies (by the global curvature rules of general relativity) perpetual equality of the absolute magnitudes of global positive energy density and global negative energy density, the magnitude of the vacuum pressure can be equated with the magnitude of critical energy density (now approximately $10^{-9} \mathrm{~J} \cdot \mathrm{m}^{-3}$ ). Remembering that Friedmann's assumptions included treatment of the cosmic fluid (i.e., the cosmic vacuum) as a perfect fluid, classical wave velocity equation (25) would seem to be appropriate for the FSC model. Thus, the cosmic wave velocity (speed of light $c$ ) should be directly proportional to the square root of the cosmic fluid bulk modulus, and inversely proportional to the square root of the cosmic fluid density. This relationship leads to Equation (27), indicating perpetual equality between the absolute magnitudes of the vacuum pressure and the vacuum energy density. So FSC predicts an equation of state $w$ term value of -1.0 exactly (assuming the convention of opposite pressure and density signages). This follows directly from the small number of FSC assumptions incorporated into the Friedmann equations. It is not an ad hoc adjustment to cosmology theory, as has clearly been the case for the various theories of cosmic inflation ([25], page 238, [26]).

In conclusion, so long as standard cosmology proponents accept, as fact that cosmic expansion is undergoing positive acceleration, however small, as implied by their claim of a 30/70 percentage ratio of total cosmic matter mass-energy to dark energy, standard cosmology is not adhering strictly to the $(\rho=p)$ stipulation of quantum field theory. To date, FSC is the only viable dark energy cosmological model which has fully-integrated general relativity and quantum features.

\section{Dedications and Acknowledgements}

Both authors dedicate this paper to the late Dr. Stephen Hawking and to Dr. 
Roger Penrose for their groundbreaking work on black holes and their possible application to cosmology. Dr. Tatum thanks Dr. Rudolph Schild of the Harvard Center for Astrophysics for his past support and encouragement. Author Seshavatharam UVS is indebted to professors Brahmashri M. Nagaphani Sarma, Chairman, Shri K.V. Krishna Murthy, founding Chairman, Institute of Scientific Research in Vedas (I-SERVE), Hyderabad, India, and to Shri K.V.R.S. Murthy, former scientist IICT (CSIR), Govt. of India, Director, Research and Development, I-SERVE, for their valuable guidance and great support in developing this subject.

\section{Conflicts of Interest}

The authors declare no conflicts of interest regarding the publication of this paper.

\section{References}

[1] Tatum, E.T., Seshavatharam, U.V.S. and Lakshminarayana, S. (2015) International Journal of Astronomy and Astrophysics, 5, 116-124.

https://doi.org/10.4236/ijaa.2015.52015

[2] Tatum, E.T., Seshavatharam, U.V.S. and Lakshminarayana, S. (2015) Journal of Applied Physical Science International, 4, 18-26.

[3] Tatum, E.T., Seshavatharam, U.V.S. and Lakshminarayana, S. (2015) Frontiers of Astronomy, Astrophysics and Cosmology, 1, 98-104.

[4] Planck Collaboration XIII. (2015) Cosmological Parameters. http://arxiv.org/abs/1502.01589

[5] Hawking, S. and Penrose, R. (1970) Proceedings of the Royal Society of London A, 314, 529-548. https://doi.org/10.1098/rspa.1970.0021

[6] Penrose, R. (1965) Physical Review Letters, 14, 57. https://doi.org/10.1103/PhysRevLett.14.57

[7] Tatum, E.T. and Seshavatharam, U.V.S. (2018) Journal of Modern Physics, 9, 1404-1414. https://doi.org/10.4236/jmp.2018.97085

[8] Tatum, E.T. and Seshavatharam, U.V.S. (2018) Journal of Modern Physics, 9, 1469-1483. https://doi.org/10.4236/jmp.2018.98091

[9] Perlmutter, S., et al. (1999) Astrophysical Journal, 517, 565-586.

[10] Schmidt, B., et al. (1998) Astrophysical Journal, 507, 46-63. https://doi.org/10.1086/306308

[11] Riess, A.G., et al. (1998) Astronomical Journal, 116, 1009-1038. https://doi.org/10.1086/300499

[12] Hawking, S. (1976) Physical Review D, 13, 191-197. https://doi.org/10.1103/PhysRevD.13.191

[13] Bekenstein, J.D. (1974) Physical Review D, 9, 3292-3300. https://doi.org/10.1103/PhysRevD.9.3292

[14] Inoue, A.K. (2004) Monthly Notices of the Royal Astronomical Society, 350, 729-744. https://doi.org/10.1111/j.1365-2966.2004.07686.x

[15] Tutusaus, I., et al. (2017). Astronomy \& Astrophysics, 602, A73. arXiv:1706.05036v1 [astro-ph.CO]. 
[16] Nielsen, J.T., et al. (2015) Marginal Evidence for Cosmic Acceleration from Type Ia Supernovae. arXiv:1506.01354v1.

[17] Wei, J.-J., et al. (2015) Astronomical Journal, 149, 102-112. https://doi.org/10.1088/0004-6256/149/3/102

[18] Melia, F. (2012) Astronomical Journal, 144, 110. arXiv:1206.6289 [astro-ph.CO]. https://doi.org/10.1088/0004-6256/144/4/110

[19] Milne, E.A. (1935) Relativity Gravitation and World Structure. The Clarendon Press, Oxford.

[20] Suzuki, et al. (2011) The Hubble Space Telescope Cluster Supernovae Survey: V. Improving the Dark Energy Constraints above $\mathrm{Z}>1$ and Building an Early-Type-Hosted Supernova Sample. arXiv.org/abs/1105.3470.

[21] Van Dokkum, P., et al. (2018) Nature, 555, 629-632. https://doi.org/10.1038/nature25767

[22] Penrose, R. (2016) Fashion Faith and Fantasy in the New Physics of the Universe. Princeton University Press, Princeton. https://doi.org/10.1515/9781400880287

[23] Verlinde, E. (2011) Journal of High Energy Physics, 4, 29-55. arXiv:1001.0785v1 [hep-th]. https://doi.org/10.1007/JHEP04(2011)029

[24] Verlinde, E. (2016) Emergent Gravity and the Dark Universe. arXiv:1611.02269v2 [hep-th].

[25] Guth, A.H. (1997) The Inflationary Universe. Basic Books, New York.

[26] Steinhardt, P.J. (2011) Scientific American, 304, 18-25. https://doi.org/10.1038/scientificamerican0411-36 